\title{
Inhalt, Vol. 17, No. 2, 1994
}

\section{Contents}

Übersichtsarbeiten

CD 44 und Metastasierung

Zöller, M., Kaufmann, M

Verfahren zur Diagnose und Stadieneinteilung beim Bronchialkarzinom als Grundlage für die

Therapie

Drings, P.

Ovarialkarzinom - wie geht es weiter?

Lahousen, $\mathrm{M}$

Review Articles

CD 44 and Metastasis

114 Zöller, M., Kaufmann, M114

Procedures for Diagnosis and Staging in Lung Cancer as a Basis for Treatment

124 Drings, $P . \quad 124$

Ovarian Carcinoma - the Way Ahead

134 Lahousen, M 134

Originalarbeiten

Klinische Phase-I-Studie mit Lobaplatin (D-19466) nach intravenöser Bolusinjektion

Fiebig, H.H., Henß, H., Mross, K., Meyberg, E,

Aulenbacher, P., Burk, K., Queißer, W.

142

Original Paper

Phase I Clinical Trial of Lobaplatin (D-19466) after Intravenous Bolus Injection

Fiebig, H.H., Henß, H., Mross, K., Meyberg, F,

Aulenbacher, P., Burk, K., Queißer, W.

142

Die unvollständige Konisation bei Carcinoma in situ der Cervix uteri

Lahousen, M., Jäger, G., Girardi, F, Pickel, H.,

Schneeweiß, W. D

150

Incomplete Conization for Carcinoma in situ of the Uterine Cervix

Lahousen, M., Jäger, G, Girardi, F, Pickel, H.,

Schneeweiß, W. D

150

Wilke, H., Preusser, P., Stahl, M., Meyer, H. I, Fink, U, 
Achterrath, W., Busche, P., Meyer, X, Harstrick, A.,

Schmoll, H.-X, Seeber, S

154

Phase II Study with Folinic Acid, Etoposide, 5-Fluorouracil and Cisplatin (FLEP) for Advanced Gastric Cancer

Wilke, H., Preusser, P., Stahl, M., Meyer, H. X, Fink, LI,

Achterrath, W., Busche, P., Meyer, X, Harstrick, A.,

Schmoll, H.-X, Seeber, $\mathrm{S}$

154

Lungentoxizität von hoch- und mittelhochdosiertem Cytosin-Arabinosid: Eine retrospektive Analyse

Dierlamm, X, Haubold-Reuter, B., Weh, H.X, Schäfer, H,

Hossfeld,D.K

158

Pulmonary Toxicity of High- and Intermediate-Dose Cytosine Arabinoside Therapy: A

Retrospective Analysis

Dierlamm, X, Haubold-Reuter, B., Weh, H.X, Schäfer, H, Hossfeld,D.K 158

Die Kombination von Grading und neuen biologischen

Faktoren (S-Phasen-Fraktion und epidermalem

Wachstumsfaktor-Rezeptor) kann Rezidiv und Überleben

von Patientinnen mit primärem, nodal negativem

Mammakarzinom prognostizieren

Kaufmann, M., v. Minckwitz, G, Finn, H. P., Schmid, H,

Goerttler, K., Bastert, G

166

Combination of Grading and New Biological Factors (S-Phase Fraction and Epidermal Growth Factor Receptor) Can Predict Relapse and Survival in Patients with Node-Negative Primary

Breast Cancer

Kaufmann, M., v. Minckwitz, G, Finn, H.P., Schmid, H,

Goerttler, K., Bastert, G

166

Durchblutungsdiagnostik maligner und benigner Tumoren des inneren Genitales

Sohn, Ch., Grischke, E. M., Kaufmann, M., Bastert, G 174

The Blood Circulation of Malignant and Benign Tumours of the Genital Interior as a Diagnostic Tool

Sohn, Ch., Grischke, E.M., Kaufmann, M., Bastert, G

Fortsetzung aufSeite 112Continued on page 112

Band 17, Heft 2, April 1994

International Journal for Cancer Research and Treatment

Inhalt 


\section{Contents}

Fax-Kommunikation

Bei akuten Leukämien und hochmalignen Non-Hodgkin-Lymphornen mit meningealem Befall sind die Galaktosyltransferase-Aktivitäten im Liquor cerebrospinalis erhöht

Ottinger, H., Cyrus, C, Belka, C, Leuner, S., Engelhard, M,

Augener, W., Brittinger, G

Unterschiedliche Anzahl von Tumorzellen im Knochenmark von Patienten mit

nichtmetastasierten kolorektalen Karzinomen

Silly, H., Wilders-Truschnig, M., Brezinschek, H.-R, Schmid, M.,

Kuss, I., Mucher, K., Stöger, H., Samonigg, H

Fax Communication

Meningeal Involvement in Acute Leukaemia and High-Grade Non-Hodgkin's Lymphoma is Associated with Elevated Activities of Galactosyltransferases in the Cerebrospinal Fluid Ottinger, H., Cyrus, C, Belka, C, Leuner, S., Engelhard, M,

180 Augener, W., Brittinger, G 180

Varying Amounts of Tumor Cells in the

Bone Marrow of Patients with Nonmetastatic Colorectal

Cancer

Silly, H., Wilders-Truschnig, M., Brezinschek, H.-R, Schmid, M.,

184 Kuss, I., Mucher, K., Stöger, H., Samonigg, H 184

Letter to the Editors

R. Hartenstein concerning:

Wilke H, et al.: Phase II study with folinic acid, etoposide, 5-fluorouracil and cisplatin (FLEP)

for advanced

Brief an die Herausgeber

R. Hartenstein zu:

Wilke H, et al.: Phase-II-Studie mit Folinsäure, Etoposid,

5-Fluorouracil und Cisplatin (FLEP) beim fortgeschrittenen

Magenkarzinom 188 gastric cancer

Wilsede-Schule 190 Wilsede-Schule

190

Pressemitteilung Press ReleaseEhrendoktortitel der Universität Basel für Thomas Karger . . 191 Honorary Doctorate Awarded to Thomas Karger

191

192

Mitteilungen onkologischer Gesellschaften194

Reports of Oncological Societies

194

Impressum 109 Imprint

Hinweise für Autoren 203 Instructions to Authors

109203 
Bibliographischer Hinweis: Inhaltsverzeichnisse dieser Zeitschrift erscheinen regelmäßig in current contents ${ }^{\circledR}$ sowie in anderen bibliographischen Diensten. 